\title{
On the Fixed-Point Implementation of Turbo Code in 3G System
}

\author{
Sun Minying \\ Institute for Communications Research, Singapore
}

Tan Wee Tiong

STMicroelectronics Asia Pacific Pte Ltd, Singapore

\begin{abstract}
Fixed-point arithmetic is mandatory in hardware implementation. This paper looks at critical issues of fixed-point arithmetic for turbo code. The techniques presented include approximation method to implement the non-linear item in Log-MAP algorithm, fixed-point representation of the soft input, soft output and internal metrics, sliding window strategy, and iterations stopping criterion. A complete set of parameters for immediate hardware implementation of turbo code is provided.
\end{abstract}

Key words: turbo code, fixed-point, $3 \mathrm{G}$

\section{INTRODUCTION}

Turbo code has attracted much attention in communications world, especially for cellular communication systems where the coding gains could be matched to bandwidth efficiency. In the standards for next generation mobile communications, turbo code is adopted as the coding scheme for both UMTS and cdma2000 system. In this paper, we discuss some critical issues of the fixed-point implementation of the decoding algorithm, in particular, the input quantization effect, bit-width of the internal metrics 
computations, the sliding window strategy, and the stopping rule. Based on simulation results, efficient solutions are proposed for real time implementation of turbo code.

Most of the applications in $3 \mathrm{G}$ systems require real time implementation. For high date rate transmission, the buffer size of the channel coding chain becomes bottleneck for the real time implementation because of the transmit time interval (TTI) based operations ( $80 \mathrm{~ms}$ maximum). For example, the maximum coded data stream size equals to $19200 * \mathrm{~N}$ bits for a $384 \mathrm{kbps}$ class user equipment (UE) capability, where $\mathrm{N}$ is the number of soft bits to represent one soft decision signal, which is determined by the decoding algorithm. It is obvious that $\mathrm{N}$ is a primary factor influencing memory requirement of the system.

Moreover, the memory size of the turbo decoding itself is also large due to the bit-wised interleaver of the coding block and bi-directional computation of path metrics requested by the algorithm. The large external memory brings out processing latency and thus lower the throughput. The number of bits used to represent soft input and internal variables is a very important design parameter that influences the total buffer size and hardware complexity. The optimisation strategies for each variable in the decoding algorithm will be discussed in details.

By using logarithm domain MAP algorithm, real time implementation of turbo code becomes feasible. However, the good performance of this coding scheme is obtained through iterative processing. Better performance can be achieved by more times of iterations, at the cost of processing latency. A stopping criterion is suggested based on the log-likelihood ratio, which is effective and simpler than the convention stopping criteria in terms of implementation.

Our discussions are limited to turbo code specified by 3GPP standard, i.e.rate $1 / 3$, constraint length 4 , with transfer function

$$
g(D)=\frac{1+D+D^{3}}{1+D^{2}+D^{3}}
$$

The code block size for the turbo code ranges from 40 to 5112 bits.

The next two sections describe the fixed-point implementation of the decoding algorithm, with Section 2 analysing different approximating methods of the non-linear item in the log-domain computation, and Section 3 discussing fixed-point representation of internal variables and proposing a simplified stopping criterion. A summary is given in Section 4. 


\section{CORRECTION ITEM IMPLEMENTATION}

In Log-MAP decoding algorithm, all the computations are linear, except the logarithm calculation, called correction item, in $\mathrm{MAX}^{*}$ operator which is described in the form:

$$
M A X^{*}\left(\delta_{1}, \delta_{2}\right)=\ln \left(e^{\delta_{1}}+e^{\delta_{2}}\right)=\max \left(\delta_{1}, \delta_{2}\right)+\ln \left(1+e^{-\left|\delta_{1}-\delta_{2}\right|}\right)
$$

There are two basic ways to approximately realize this non-linear operation in fixed-point arithmetic - look up table and linear approximation. A fixed-point number is represented by $(d, f)$ with $d$ a total bit-width and $f$ the fraction part. The non-linear function $f(x)$ is then transformed to $2^{f} \times f(x$ $/ 2^{f}$ ). Generally 3 bit precision, i.e. $f=3$ is sufficient to achieve as good performance as infinitely soft quantization [5]. The least square method was used to approximate the natural logarithm function, and the fixed-point linear function for 3 bit precision was obtained in the form :

$$
f_{f i x}(x)=\left\{\begin{array}{lr}
5-x / 4, & 0 \leq x \leq 16 \\
0, & x>16
\end{array}\right.
$$

The look up table method is described in details in [5]. The two methods are identical in terms of performance as well as computation complexity. What attracts us most is a constant approximation method, i.e. the correct item is approximated by a constant value. For a 6-bit input quantization, we select value 3 from look up table to approximate the correction item. The BER performance is shown in figure 1. It indicates that the constant approximation method has less than $0.05 \mathrm{~dB}$ loss comparing with look up table method. On the other hand, the decoding algorithm is sensitive to the SNR estimation because of the nonlinearity computation. The performance of each implementation method as a function of SNR estimation offset is displayed in figure 2. It is found that MAX-Log-MAP is not sensitive to the SNR estimation accuracy as expected. In contrast, the other three methods are sensitive to SNR estimation error. And the constant approximation behaves similarly as the other two methods in the presence of estimation error. 


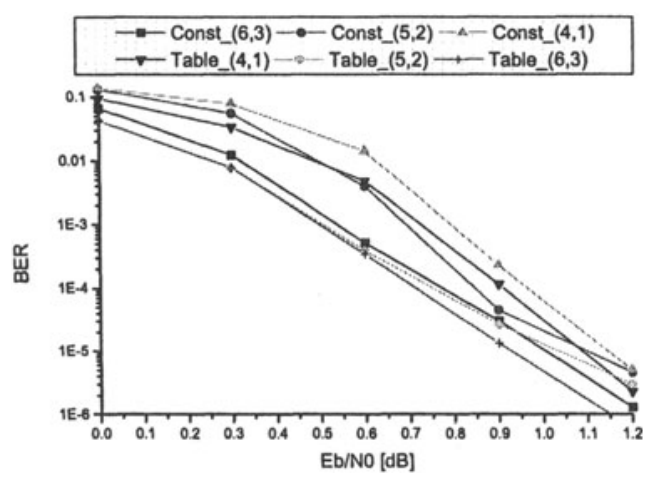

Figure 1 Performance comparison of different correction item implementation for various quantization level

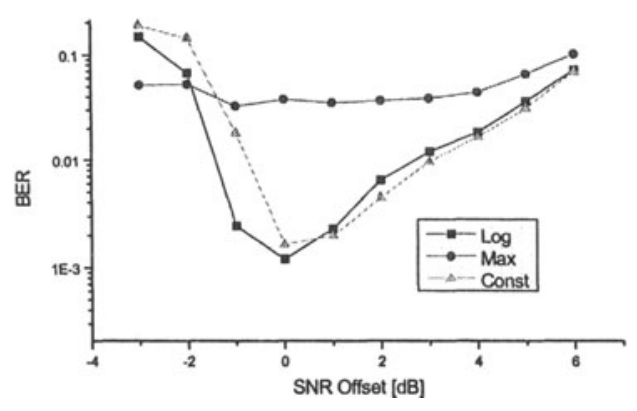

Figure 2. SNR mismatch effect at $0.5 \mathrm{~dB}$

From these simulation results, we can conclude that the constant approximation Log-MAP algorithm is a good solution with 6-bit quantized soft input. However, if the number of soft input is reduced to 4-bit width. The performance of constant approximation method approaches MAX-LogMAP algorithm with $0.5 \mathrm{~dB}$ loss of $\mathrm{E}_{\mathrm{b}} / \mathrm{N}_{0}$. The look up table method of 4-bit quantization losses less than $0.1 \mathrm{~dB}$ comparing with 6-bit quantization. In $3 \mathrm{G}$ system, Viterbi decoder generally co-exist with turbo decoder. 4-bit soft input is sufficient for Viterbi decoder to get desirable performance. Therefore, to save memory budget, 4-bit quantization is a better solution for $3 \mathrm{G}$ applications.

To reduce the storage of path metrics, sliding window (SW) technique was introduced to the forward and backward state metrics computation for MAP decoder. It is extremely useful to reduce the working memory. The forward and backward metrics are computed with a shortened window size $\mathrm{N}_{\mathrm{s}}=\mathrm{N} / \mathrm{W}$, where $\mathrm{N}$ is the entire block size and $\mathrm{W}$ is the number of 
segmentations. The SW technique can reduce buffer size of the backward metrics by $\mathrm{W}$ times. The disadvantage of SW technique is that a training sequence is necessary for the backward metrics computation in order to achieve reliable distribution of states for processing since the initial states of the backward metrics are unknown for all shortened blocks except the last one. Total redundant backward computations to decode one block of data are $\mathrm{N}_{\mathrm{b}} *\left(\mathrm{~N}_{\mathrm{s}}-1\right)$. Therefore, $\mathrm{N}_{\mathrm{s}}$ shall be deliberately selected to compromise between memory size and computation overhead. According to the simulation results, $\mathrm{N}_{\mathrm{b}}=6 *(\mathrm{~m}+1)$ is sufficient to get reliable state distribution of $\beta$. In our implementation, the window size is 100 bits and $N_{b}$ is 24 bits. For the worst case in 3GPP standard, the code block size for turbo code is 5114 , the SW scheme saves one third of the total memory while the computation increases $8 \%$ per iteration.

\section{INTERNAL VARIABLES AND STOPPING RULE}

The maximum difference between different forward state metrics can be loosely estimated as maximum difference between branch metrics. With the decoding block size increase, forward path metrics $\alpha$ and backward path metrics $\beta$ will increase without bound. The magnitudes of $\alpha$ and $\beta$ increase along with the recursive processing and the magnitude of LLR increase with the number of iterations. Subtraction is commonly used for normalisation because the soft output is only influenced by the difference between state metrics instead of their absolute values. At a given time $k$, the maximum metric is determined and subtracted from $\alpha$ or $\beta$ at each state to scale down the values. The number of bits for each variable is then determined by observing its value range which is shown in Figures 3 and 4.

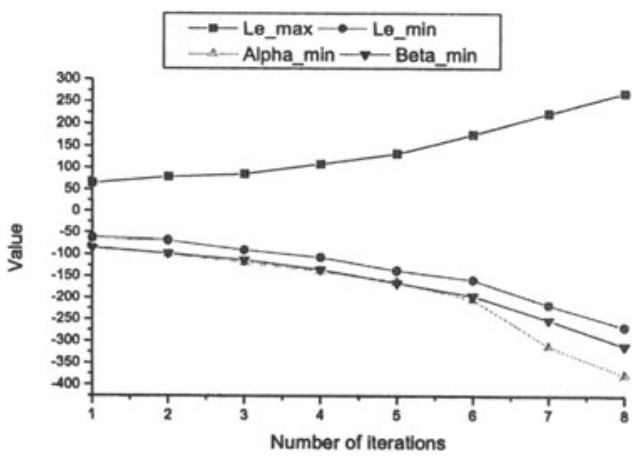

Figure 3 Value range of internal variables vs. number of iterations 


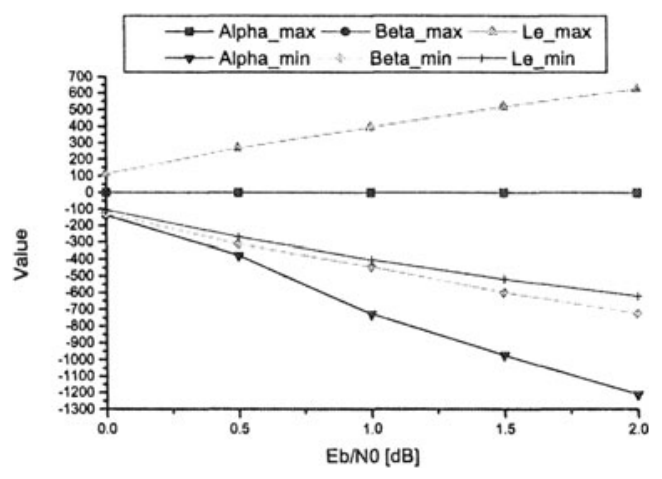

Figure 4 Value range of internal variables vs. $\mathrm{E}_{\mathrm{b}} / \mathrm{N}_{0}$

According to the value range, 12-bit width is sufficient to contain all the received information during the computation. It is found that when the clipping level is reduced from 12 bits to 8 bits, the performance degradation due to quantization distortion is less than $0.1 \mathrm{~dB}$. It is interpreted that, when the value of LLR reaches a certain level, reliable hard decision can be made upon LLR and truncation errors will not influence the performance.

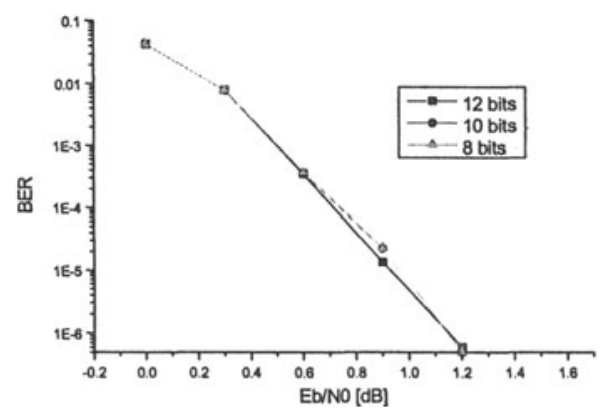

Figure 5 Performance of variable bit-width representation of path metrics.

Moreover, the LLR trend tells us that after the LLR value reaching its clipping level, e.g. 127 for 8-bit presentation, further computation of LLR is not necessary because the value is overflow and will be truncated to the maximum value. A simplified stopping criterion is suggested based on this numerical property of LLR. If $\min |L L R|>$ threshold, the LLR is considered reliable enough for a hard decision. Following this stopping rule, the iteration can stop at any decoding stage for a particular iteration as long as the criterion is satisfied. The advantage of our LLR stopping criterion over 
conventional methods relies on the immediate halting at the current iteration, instead of waiting for comparison of two LLR results. The efficiency of this method is shown in figures 6 and 7 , which indicates that the average number of iterations to achieve a certain BER performance is significantly reduced.

The dependence of the value range on the $E_{b} / N_{0}$ is presented previously in figure 4. The threshold is selected according to the value range of LLR in terms of following conditions: If the threshold is chosen based on value range at low $E_{b} / N_{0}$, the iteration may halt before a reliable LLR is achieved at high $\mathrm{E}_{\mathrm{b}} / \mathrm{N}_{0}$ and consequently, the performance is largely discounted. If the threshold is chosen based on high $E_{b} / N_{0}$ value range, extra effort is spent to satisfy the criterion at low $E_{b} / N_{0}$. For example, At $3 \mathrm{~dB} \mathrm{E}_{\mathrm{b}} / \mathrm{N}_{0}, 4$ iterations are needed to accumulate a LLR value of 311 , as compared to 18 iterations needed to reach the same LLR level at $0.3 \mathrm{~dB}$. Actually, the processing latency of 18 iterations is not acceptable for real time implementation Therefore, the threshold shall be adaptively selected for different $E_{b} / N_{0}$. In our implementation, the threshold is chosen based on high $\mathrm{E}_{b} / \mathrm{N}_{0}$ value range, and at the same time, maximum number of iterations is fixed for low $E_{b} / N_{0}$. The strategy is a compromising of performance and implementation complexity.

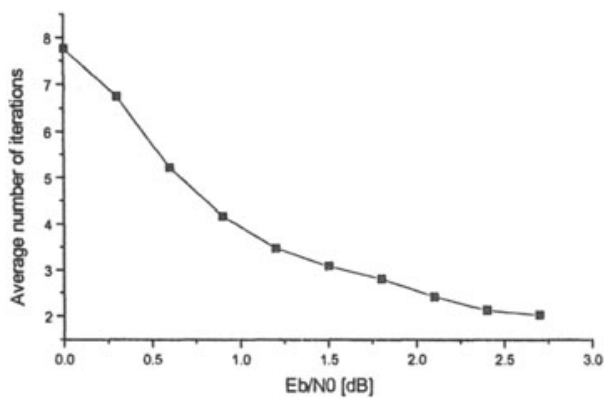

Figure 6 Average number of iterations vs. $E_{b} / N_{0}$ 


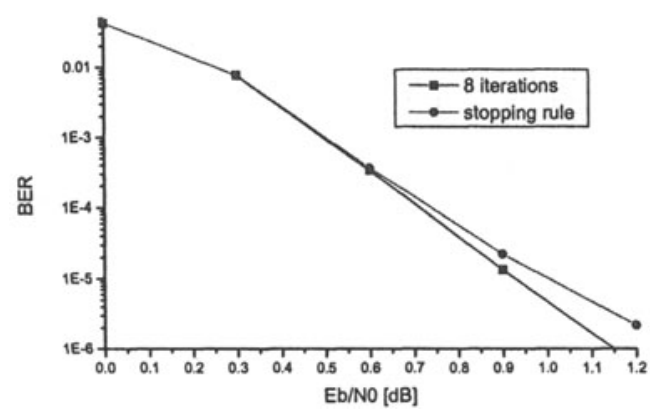

Figure 7 BER performance of the proposed stopping rule.

\section{CONCLUSIONS}

The optimisations for fixed-point implementation presented in this paper lead to significant reduction in hardware resource. By reducing the soft input quantization to 4-bit, the buffer size for TTI based operation is saved by $33 \%$. Moreover, by using the $(100,24)$ sliding window scheme, the state metrics memory only equals to window size which saves around one third of the storage for turbo decoding of large coding blocks. The clipping and stopping method can reduce the computation by $50 \%$ at high $\mathrm{E}_{\mathrm{b}} / \mathrm{N}_{0}$. If the clipping and stopping method is applied to $\alpha$ computation, the computation of each iteration can be further reduced by $20 \%$.

\section{REFERENCES}

[1] Berrou, A. Glavieux, and P. Thitimajshima, Near Shannon limit error-correcting coding and decoding, Proceeding 1993 International Conference on Communication, p.10641070.

[2] L.R.Bahl, J.Cocke, F.Jelinek, J.Raviv, “Optimal Decoding of Linear Codes for Minimizing Symbol Error Rate”, IEEE Trans. Inf. Theory, Vol. IT-20, March 1974, pp.284-287.

[3] S. Wilson, Digital Modulation \& Coding, Prentice-Hall, 1996, p. 604.

[4] A.J. Viterbi, "An intuitive justification of the MAP decoder for convolutional codes", IEEE JSAC, vol. 16, pp. 260-264, Feb. 1998

[5] G.Montorsi, and S. Benedetto, "Design of Fixed-Point Iterative Decoders for Concatenated Codes with Interleavers", IEEE JSAC, vol. 19, No.5, May 2001.

[6] 3GPP TS 25.212 version 3.4.0, "Multiplexing and channel coding (FDD)",

[7] R.Y.Shao, S. Lin , "Two Simple Stopping Criteria for Turbo Decoding," Electronic Letters, vol.35, pp. 701-702, 1999. 